\title{
Editorial
}

\section{Glucose, insulin, and the cardiovascular system}

There is increasing evidence to support the beneficial effects of glucose-insulin-potassium infusions (GIK) in acute myocardial infarction, cardiogenic shock, and cardiac surgery. An article in this issue suggests some benefits may also accrue during the treatment of chronic heart failure. ${ }^{1}$

\section{Background physiology}

The use of GIK to improve ischaemic cardiac dysfunction has been based on two principles. First, insulin stimulates myocardial $\mathrm{Na}^{+} \mathrm{K}^{+}$ATPase, increasing reuptake of $\mathrm{K}^{+}$, stabilising the cell membrane, and reducing the incidence of dysrrhythmias. Second, insulin stimulates the myocardial uptake of glucose, increasing the provision of intracellular substrate. However the significance of the relatively small increase in ATP, which GIK produces by enhancing anaerobic oxidation of glucose, has been questioned. The evidence for attenuation of ischaemia induced reduction in ATP stores is scanty. Nevertheless, recent work suggests that the position of the glycolytic enzymes within the cell may ensure that the little ATP that is produced is situated in regions critical to the maintenance of cellular membrane functions such as calcium and sodium homeostasis. ${ }^{2}$ Provision of a high glucose substrate protects myocytes from the toxic effects of the increase in intracellular calcium induced by ischaemia. ${ }^{3}$ Previous concerns that during ischaemia these benefits may be limited by the generation of inhibitors of glycolysis, such as lactate, have been allayed by recent studies of myocardial infarction showing that the infarct area is a region of low flow, not zero flow, sufficient to deliver substrate and remove lactate. This provides a rationale for the use of GIK in acute ischaemia.

Other metabolic effects of ischaemia are also important, not least the increased production of catecholamines which leads to glucose intolerance and increased concentrations of free fatty acids (FFA). ${ }^{4}$ Cardiac muscle preferentially utilises FFA, which produce more ATP per carbon atom than glucose metabolism, but demands more oxygen to do so. However the high concentration of FFA produced during ischaemia can generate dysrrhythmias, depress the myocardium, ${ }^{5}$ and may endanger the recovery of ischaemic but viable tissue. ${ }^{4}$ Insulin has an anti-FFA effect, which may be particularly important when the toxic effects of FFA are accentuated by catecholamines. ${ }^{6}$

Insulin resistance syndrome (IRS) is closely linked with the development of cardiac disease. IRS is characterised by glucose intolerance, hyperinsulinaemia, dyslipidaemia, obesity, and hypercoagulability. ${ }^{7}$ Initially, serum glucose is maintained within the normal range but may progress to overt diabetes, but even in those who do not, hyperinsulinaemia remains. Hyperinsulinaemia induces overexpression of the angiotensin 1 receptor on endothelium, which mediates the biological actions of the renin-angiotensin system such as vasoconstriction and cellular proliferation. This enhancement of the action of angiotensin II may explain the link between hyperinsulinaemia and hypertension, and subsequent atherosclerosis and coronary heart disease. These cardiovascular actions may be augmented by the effects of insulin on the sympathetic nervous system and the L-arginine-nitric oxide pathway. Given this myriad of pathophysiological actions of insulin, it would not be unreasonable to suppose that insulin may have a beneficial effect on the long term management of congestive cardiac failure.

\section{Clinical studies in myocardial infarction}

Sodi-Pallares and colleagues first showed a beneficial effect of GIK infusions administered to patients with acute myocardial infarction in $1962 .{ }^{\circ}$ Failure to reproduce these results led to the use of GIK being abandoned until the 1980s when Rackley and associates defined the optimal dose of insulin required to suppress FFA, and confirmed the improvement in infarct patients. ${ }^{9}$ In 1997 a metaanalysis showed a reduction in mortality of $28 \%$, equivalent of 49 lives saved per 1000 patients treated. ${ }^{10}$

A prospective, randomised study of GIK in over 400 patients with myocardial infarction showed a $66 \%$ reduction of in hospital mortality for GIK and reperfusion compared to reperfusion alone. ${ }^{11}$ Even though the average time from onset of acute myocardial infarction symptoms to initiating treatment was 10-11 hours, the combined end point of death, severe heart failure, and non-fatal ventricular fibrillation was reduced by $40 \%$. Two GIK regimens were used, a high dose (previously shown to maximally suppress FFA concentrations and myocardial uptake of FFA) and a low dose. Although the in-hospital results were similar between the treatment groups, only the high dose GIK group had a significant survival advantage at one year. This is consistent with the results of the meta-analysis, which predicted a decrease in mortality risk of $48 \%$ when only trials using the high dose were considered, compared to only $28 \%$ when both high and low dose trials were included. ${ }^{10}$

\section{Clinical studies in cardiac surgery}

Cardiac surgery produces a period of myocardial ischaemia followed by reperfusion. Interpretation of the few trials available are handicapped by the poor design, small size, the GIK dose chosen, and its timing in relation to the ischaemic insult, and the outcomes studied. However, they do tend to show a similar pattern of benefit with increases in cardiac index, decreased inotropic and mechanical support, and a reduction in dysrrhythmias. For example, in a randomised, prospective study of urgent coronary artery bypass grafting for unstable angina, GIK was given before bypass and for 12 hours afterwards. The GIK group had a notably greater cardiac index with less inotropic support, and the incidence of perioperative atrial fibrillation was dramatically reduced. ${ }^{12}$ Another blinded, controlled study showed GIK started before bypass produced a pronounced improvement in cardiac index, with the greatest effect in those patients with the worst left ventricular function. ${ }^{13}$ Taegtmeyers' group also showed that GIK greatly decreases FFA concentrations while increasing cardiac index by approximately $40 \%$ without change in inotropic dosages in post-bypass cardiogenic shock. In contrast the cardiac index remained unchanged in the control group despite doubling of the inotropic support. In a larger follow up 
study, 322 patients with pump failure post-bypass were treated with GIK, reducing in hospital mortality by one third and length of intensive care and hospital stay. ${ }^{14}$

\section{Clinical studies in congestive cardiac failure}

There are good theoretical grounds on which to suppose that GIK may be beneficial, not least because insulin resistance is an important factor in congestive cardiac failure. $^{15}$ Animal work suggests that hypertrophied and volume overloaded hearts are more sensitive to ischaemia, have impaired myocardial energy reserves, increased susceptibility to acute metabolic stress, and therefore derive a greater protective effect from GIK. ${ }^{16}$ As yet there have been no worthwhile clinical trials of GIK in congestive cardiac failure.

That insulin produces skeletal muscle vasodilatation has been known since 1990, although the mechanism has yet to be elucidated. Parsonage and colleagues have demonstrated this effect of insulin in chronic heart failure. ${ }^{1}$ They have shown a redistribution of cardiac output to skeletal muscle with an essentially unchanged cardiac output induced by administering insulin at a rate equivalent to approximately 5-10 units/hour in a $70 \mathrm{~kg}$ man using a euglycaemic clamp. This exceeds the high dose infusions used in the infarction studies (approximately 5 units/ hour $^{11}$ ), so it is surprising that there is such little direct myocardial effect. However, the fall in heart rate suggests an increase in stroke volume, which may represent an improvement in contractility mediated by insulin.

This paper raises several questions. Do the effects of the euglycaemic clamp reflect the clinical administration of GIK? Do the increases in skeletal muscle blood flow correlate with increased exercise capacity of the limb, and more importantly of the patient as a whole? If there is a benefit, how long does it last? If only as long as the infusion, then the therapeutic potential is limited. What are the myocardial effects of insulin infusions in congestive cardiac failure? Is there a role for GIK in acute exacerbations of congestive cardiac failure, particularly when a precipitating factor can be identified and recovery may be expected? Will an inotropic effect be associated with an increased mortality, as it was for phosphodiesterase inhibitors? If not, is there a role for insulin in the longer term treatment of congestive cardiac failure, particularly since newer methods of insulin administration such as inhalers will soon be available?

\section{Conclusions}

The use of GIK infusions in medical and surgical ischaemia is a safe and cheap technique with proven benefits. Generally insulin infusion rates are 5-10 units/ hour, with serum glucose maintained between 5-10 $\mathrm{mmol} / 1$ and potassium $4-5 \mathrm{mmol} / \mathrm{l}$. Frequent monitoring of glucose and potassium will ensure safety, and should be continued for 24 hours after the infusion is stopped to ensure that rebound hyperkalaemia, and possible cardiac arrest, is avoided. Further work will answer some of these outstanding questions, including its role in congestive cardiac failure.

\section{J BROOMHEAD} M P COLVIN

Department of Cardiothoracic Anaesthesia, London Chest Hospital, Bonner Road, London E2 97X, UK

peter.colvin@royalhos-tr.nthames.nhs.uk

1 Parsonage W, Hetmanski D, Cowley A. Beneficial haemodynamic effects of insulin in chronic heart failure. Heart 2001;85:508-13.

$2 \mathrm{Xu} \mathrm{K}$, Zweier J, Becker L. Functional coupling between glycolysis and sarcoplasmic reticulum calcium transport. Circulation Res 1995;77:88-97.

3 Kondo R, Apstein C, Eberli F, et al. Increased calcium loading and inotropy without greater cell death in hypoxic rat cardiomyocytes. Am f Physiol 1998;275:H2272-82.

4 Opie L. Metabolic response during impending myocardial infarction, 1: relevance of studies of glucose and fatty acid metabolism in animals. Circulation 1972;45:483-90.

5 Oliver M, Opie L. Effects of glucose and fatty acids on myocardial ischaemia and arrhythmias. Lancet 1994;343:155-8.

6 Gal J, Smith A, Riedel B, et al. Preservation and protection of myocardial function. F Cardiothor Vasc Anesth 2000;14(suppl 1):22-36.

7 Garvey W, Hermayer K. Clinical implications fo the insulin resistance syndrome. Clinical Cornerstone 1998;1:13-28.

8 Sodi-Pallares D, Testelli M, Fishleder B, et al. Effects of an intravenous infusion of a potassium-glucose-insulin infusion on the electro-cardiographic signs of myocardial infarction. Am f Cardiol 1962;9:166-81.

9 Rackley C, Russell R, Rogers W, et al. Clinical experience with glucose-insulin-potassium therapy in acute myocardial infarction. Am Heart f 1981;102:1038-49.

10 Fath-Ordoubadi F, Beatt K. Glucose-insulin-potassium therapy for treatment of myocardial infarction: an overview of randomized placebotreatment of myocardial infarction: an over

11 Diaz R, Paolasso E, Piegas L, et al. Metabolic modulation of acute myocardial infarction. Circulation 1998;98:2227-34.

12 Lazar H, Phillipides G, Fitzgerald C, et al. Glucose-insulin-potassium solutions enhanced recovery after urgent coronary artery bypass grafting. $\mathcal{F}$ Thoracic Cardiovasc Surg 1997;113:354-62.

13 Girard C, Quentin P, Bouvier H, et al. Glucose and insulin supply before cardiopulmonary bypass in cardiac surgery: a double blind study. Ann Thorac Surg 1992;54:259-63.

14 Taegtmeyer H, Goodwin G, Doenst T, et al. Substrate metabolism as a determinant for postischaemic functional recovery of the heart. $A m \mathcal{F}$ determinant for postischac

15 Swan J, Walton C, Godsland I, et al. Insulin resistance in chronic heart failure. Eur Heart f 1994;15:1528-32.

16 Apstein C. Metabolic approaches in ischaemic heart disease. Eur Heart $\mathcal{f}$ 1999;1(suppl O):O1-O10. 